\title{
New Apparatus for Determination of Size Distribution of Particles in Fine Powders
}

\author{
Robert T. Knapp, California Institute of Technology, Pasadena, Calif.
}

$\mathrm{E}$ VERY year a greater need for accurate analysis of the size distribution of subsieve material is being felt by the engineer, because of the increasingly vital part it plays in the manufacture of cement, pigments, and other powdered products. Sieves are a satisfactory means of obtaining this information as long as the material is relatively coarse, but when from 70 to 95 per cent of the sample passes the 200-mesh screen (the finest one giving consistent reading), some new scheme of analysis must be used.

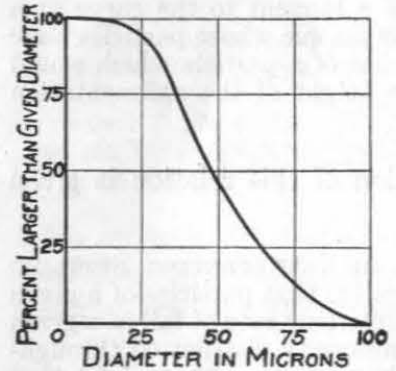

Figure 1. Cumulative Distribution Curve
Several methods of analyzing this fine fraction have been developed. For example, Professor Work at Columbia has perfected an excellent microscope technic. Elutriation and sedimentation methods employing various fluid media have been shown to be feasible, and additional methods are constantly being developed, but most of them are slow and tedious.

Some time ago the Riverside Cement Company inaugurated a comprehensive research program. A consideration of its'scope quickly showed that to carry on the program it was absolutely necessary to have some reliable method of making large numbers of subsieve analyses. After considerable study it was concluded that none of the existing instruments were satisfactory for the purpose; therefore, the development about to be described was undertaken.

Before starting the design of the instrument a careful analysis of the needs of the research program was made, which resulted in setting up the following five specifications: - -

1. High accuracy.

2. Ability to determine complete size distribution as distinguished from a fineness modulus or a value of total surface.

3. Capacity for analyzing a

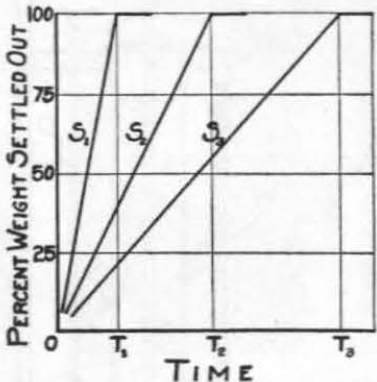

Figure 2. Setruing Curve large number of samples per day.

4. Ability to use relatively large samples to reduce sampling errors.

5. Freedom from personal equation of the operator.

The principle upon which the new instrument was to operate was then chosen by comparing the above specifica- tions with the operating characteristics of existing instruments.

The microscopic method was eliminated because it was too slow and employed too small a sample. Elutriators appeared unsuitable because of their doubtful accuracy and the length of time required to separate the sample into a large number of fractions. Methods involving light-scattering appeared to show promise for the determination of total surface but not for size distribution. In spite of the fact that existing instruments required prohibitive amounts of attention and their results were largely qualitative, the sedimentation method appeared to be the most promising; consequently this principle was adopted as the basis of the new design.

\section{THEORY}

Stokes' LAw. When a small body is allowed to fall freely in a viscous field, it soon reaches a velocity where the downward acceleration is balanced by the friction. Therefore, the velocity ceases to increase. This limiting velocity is expressed by the equation known as Stokes' law

$$
V=\frac{2 g\left(\sigma-\sigma_{m}\right) r^{2}}{9 \eta}
$$

where $V=$ velocity of fall

$$
\begin{aligned}
& g=\text { acceleration of gravity } \\
& \sigma=\text { density of falling substance } \\
& \sigma_{m}=\text { density of fluid medium } \\
& \eta=\text { viscosity of fluid medium } \\
& r=\text { radius of the particle }
\end{aligned}
$$

For the present purpose, the time required for a particle to fall a given distance is more interesting than the velocity. Therefore, the equation becomes

$$
T=\frac{H}{\bar{V}}=\frac{9 H_{\eta}}{2 g\left(\sigma-\sigma_{m}\right) r^{2}}
$$

where $T=$ time of fall

$$
H=\text { height of fall }
$$

If the height, viscosity, and densities are held constant, this becomes

$$
T=\frac{K}{r^{2}} \text { or } r=\frac{K_{1}}{\sqrt{\bar{T}}}
$$

$K$ and $K_{1}$ being constants.

If a known weight of material composed of different sized particles is allowed to settle a distance $H$ through a column of liquid, the relation between the weight of the material reaching the bottom and the time can be determined. By means of Equation 3, values of the radius, $r$, or diameter, $d$, can be substituted for the corresponding times, $T$, and the curve similar to Figure 1 constructed.

This curve supplies the desired information about the size distribution of the particles in the sample. Unfortunately, 
there are great difficulties in the experimental determination of this curve. The most serious one is encountered when an attempt is made to start a sample settling from the top of the column of liquid. Since the layer of sample (dry or

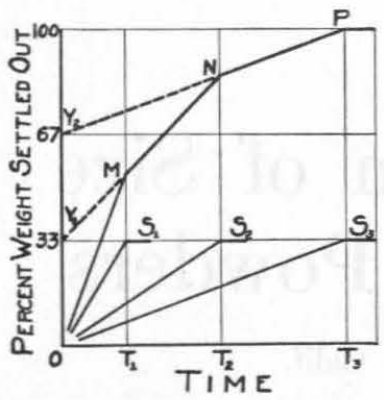

Figure 3. Settuing Curve mixed with a small quantity of liquid) is considerably more dense than the underlying liquid, the condition is very unstable and the sample tends to go en masse to the bottom, forming strong eddy currents on the way. This is a fundamental difficulty, and one for which as yet no remedy has been found.

Operation of Sedimentation Apparatus (3). The simplest method of starting sedimentation is to stir the sample thoroughly into the liquid until a homogeneous mixture is obtained, and then allow it to settle. However, this entirely changes the relation between the time of settling and the amount of material reaching the bottom, because the height is no longer the same for each particle. Nevertheless, there is a definite relation between the rate of settling in this latter case and that of the hypothetical sample which is all started from one definite height. Therefore, if the time settling rate relation is determined for the homogeneous mixture, the size distribution of the particles in the sample is readily obtainable. This relation is unique and interesting and is best illustrated by the consideration of a hypothetical example.

A sedimentation column of height $H$ is available. Three samples of known uniform size are to be used: The first sample, $s_{1}$, consists of particles, all of which have a radius $r_{1}$; the second, $s_{2}$, of radius $r_{2} ;$ and the third, $s_{3}$, of radius $r_{3}$. All the constants of Equations 2 and 3 are known. Substituting in the numerical values, it is determined that the particles of $s_{1}$ will settle distance $H$ in $T_{1}$ minutes, $s_{2}$ in $T_{2}$, and $s_{3}$ in $T$.

First $s_{1}$ is placed in the sedimentation column, mixed thoroughly, and allowed to settle. Since the particles are all of the same size, they will all settle with the same velocity. As they are distributed uniformly throughout the column, the amount collected on the bottom will be a linear function of the time, being 0 per cent at time 0 , and 100 per cent at the time $T$, as shown in Figure 2, curve $s_{1}$. Likewise, if sample $s_{2}$ is tested in the same manner the result will be represented by curve $s_{2}$, and in the same way $s_{3}$ is obtained. All these relations are straight lines, because all the samples consisted of uniform sized particles, which were uniformly distributed in the sedimentation chamber.

Now, a sample consisting of one-third $s_{1}$, one-third $s_{2}$, and onethird $s_{3}$ is placed in the sedimentation column. If $s_{1}$ were settling out alone it is obvious that the curve would be the one labeled $s_{1}$ in Figure 3, since there is only $33^{1} / 3$ per cent of $s_{1}$. Likewise $s_{3}$ and $s_{3}$ would be as shown, if either were the only one present.

Since they are all settling together, in the time $T_{1}$, all of $s_{1}$ will be out, together with $a$ per cent of $s_{2}$ and $b$ per cent of $s_{3}$; therefore, the total per cent out will be:

but

$$
M=331 / s+a+b
$$

Therefore

$$
\begin{gathered}
a=331 / 2 \times \frac{T_{1}}{T_{2}} \text { and } b=331 / 2 \times \frac{T_{1}}{T_{2}} \\
M=331 / 2\left(1+\frac{T_{1}}{T_{2}}+\frac{T_{1}}{\bar{T}_{2}}\right)
\end{gathered}
$$

Likewise at time $T_{2}$ all of $s_{1}$ and $s_{2}$ will be out, together with

That is, $\quad \begin{aligned} c & =331 / 3 T_{2} / T_{2} \text { from } s z \\ N & =331 / 3\left(1+1+T_{2} / T_{3}\right)\end{aligned}$

At time $T_{2}$, all the sample will have settled out, so point $P=$ 100 per cent. Since the individual settling curves are straight lines, the composite curve will be the broken straight line $O-M-N-P$. It is instructive to investigate where $N-M$ produced crosses the $y$ axis-i. e. the point $Y_{1}$. By inspection this simple geometric relation is obtained:

$$
Y_{1}=M-T_{1}\left(\frac{N-M}{T_{2}-T_{1}}\right)
$$

Substituting in the previously determined values for $M$ and $N$ and simplifying, this becomes $Y_{1}=33^{1 / 3}$.

Likewise, $P-N$ produced intersect the $y$ axis at $Y_{2}$

$$
\begin{aligned}
Y_{2} & =N-T_{2}\left(\frac{P-N}{T_{2}-T_{2}}\right) \\
& =662 / 3
\end{aligned}
$$

This is significant, since $33^{1} / 3$ is the per cent of $s_{1}$ in the total sample, and $66^{2} / \mathrm{s}$ is the sum of the percentages of $s_{1}$ and $s_{2}$.

Oden's Interpretation of Settring Curves. The above hypothetical example serves to illustrate the method used by Oden to interpret settling curves. He proved that the above relations hold in any suspension of different sized particles of the same substance where the sizes are such that they follow Stokes' law. A general statement of the method is as follows:

If the sedimentation curve is plotted with the time of the settling as abscissa and the weight of the material as ordinate, then the intercept on the $y$ axis of a tangent to the curve at a point $T$ will give the per cent of the sample whose particles have diameters equal to or larger than that of a particle which would just settle a distance equal to the height of the sedimentation column in the time $T$.

A more mathematical derivation of this relation is given below.

Proof of Intercept Method of Interpreting Setrling CuRves. The derivation postulates $(a)$ that particles of a given diameter have a constant and characteristic rate of fall in a given liquid; (b) that the particles are uniformly distributed throughout the liquid at the instant settling commences; and (c) that the particle concentration is sufficiently low so that there is no interference.

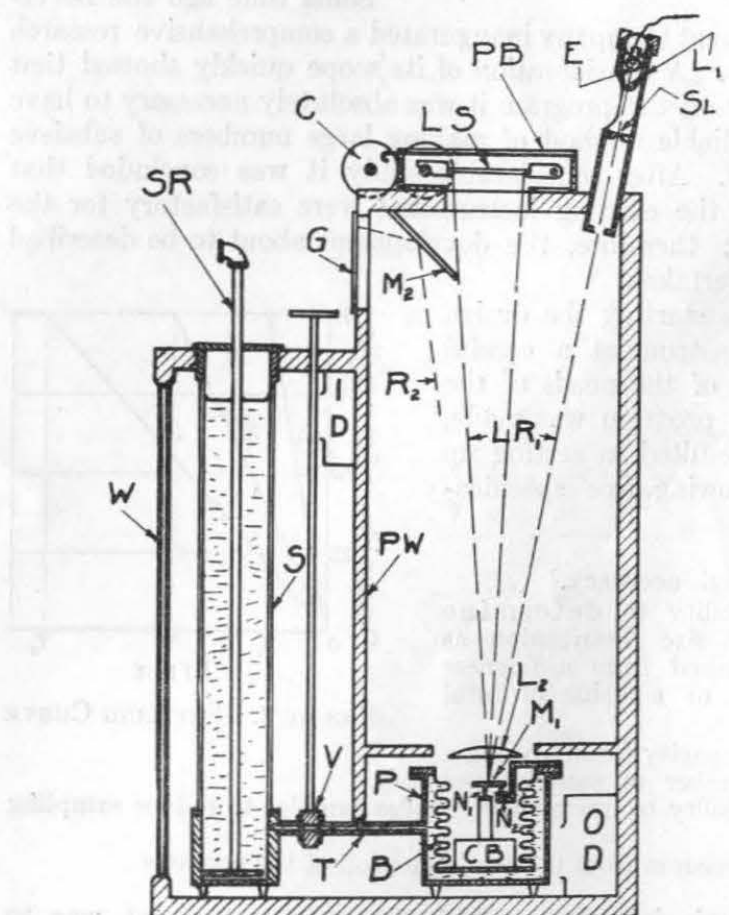

Figure 4. Sectional Diagram of MicroneTER UNIT 
Given a settling curve of weight settled out, $W$, plotted as a function of time $t$, let $W_{t}$ equal the weight of the fraction whose particles have a fall time $t$ for the height of the liquid column.

Therefore $\frac{W_{t}}{t}$ equals the rate of settling out of this fraction and $\tau \frac{W t}{t}$ equals the weight of this fraction settled out in time $\tau$.

Now $\frac{d W}{d t}-\int_{t}^{\infty} \frac{W_{t}}{t} d t$, since all fractions with settling times of $t$ or less are all settled out and therefore do not contribute to $\frac{d W}{d t}$.

Consider time $\tau$ :

$$
\begin{aligned}
W_{\tau} & =\int^{\tau} W_{t} d t+\int_{\tau}^{\infty} \tau \frac{W_{t}}{t} d t \\
& =\int_{0}^{\tau} W_{t} d t+\tau \int_{\tau}^{\infty} \frac{W_{t}}{t} d t
\end{aligned}
$$

That is, total weight settled out at time $\tau=$ the sum of all fractions having settling times of $\tau$ or less + proportional amounts of all fractions having settling times greater than $\tau$.

Now

$$
\left[\frac{d W}{d t}\right]_{\tau}=\int_{\tau}^{\infty} \frac{W_{t}}{t} d t
$$

Substituting in Equation 4

$$
\text { or } \begin{aligned}
W_{\tau} & =\int_{0}^{\tau} W_{t} d t+\tau\left[\frac{d W}{d t}\right]_{\tau} \\
\int_{0}^{\tau} W_{t} d t & =W \tau-\tau\left[\frac{d W}{d t}\right]_{\tau} \\
& =I \tau
\end{aligned}
$$

since $\int_{\tau}^{\tau} W_{t} d t$ is the total weight of all fractions having settling times of

It should be noted that this derivation assumes no specific law of settling beyond the three postulates; therefore this method of analysis may be used under any settling conditions for which the relation between particle diameter and settling time can be experimentally determined, whether or not Stokes' law is applicable.

Measuring Amount of Material Setrtling Out. The next question to be answered is: How can the amount of material reaching the bottom of the sedimentation column in a given time be measured? There are several ways of doing this, but one of the simplest is to measure the hydrostatic pressure just above the bottom of the column. This is the method used by Wiegner in his instrument for silt analysis (4). Since the particles are neither accelerating nor decelerating, their entire weight must be supported by the liquid and they can be considered as if they were stationary and suspended in the liquid. Obviously, in the latter case

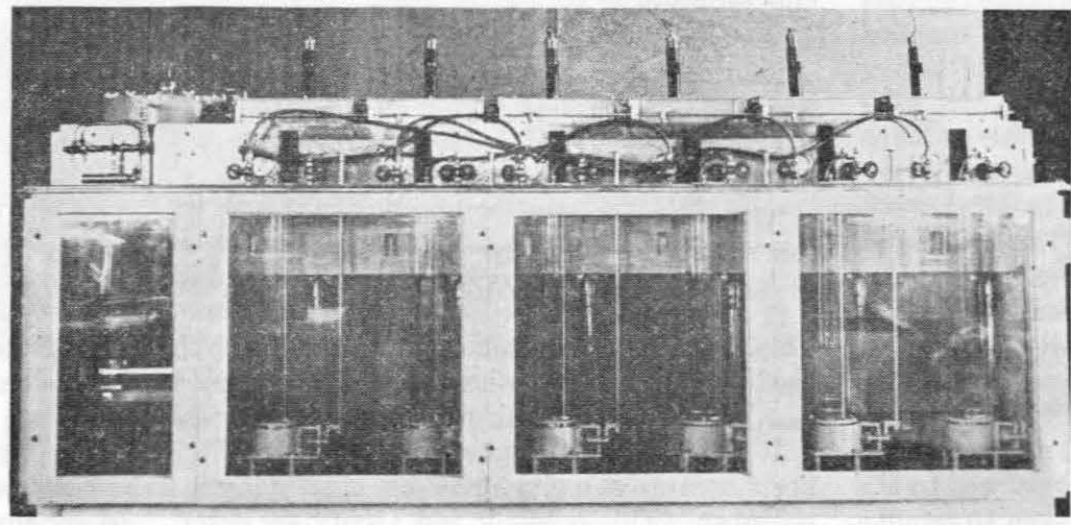

Figure 5. Photograph of Apparatus before Installation

the liquid because a volume of liquid equal to the volume of the settled-out material flows into the column. Thus it can be stated that the change in pressure at the point on the wall of the sedimentation column is directly proportional to the amount of material that has settled past this point.

Since sedimentation instruments operate on the assumption that the suspended particles obey Stokes' law, a study of Equation 1 and the range of its validity brings out the following as conditions which must be fulfilled if accurate results are to be obtained:

1. The viscosity of the fluid must remain constant.

2. The densities of both the fluid and the material under test must not vary.

3 . The relative velocity between the fluid and the fastest moving particles must not be over a certain critical maximum-i, e., the velocity above which local turbulence is set up.

4. There must be no thermal convection currents to upset equilibrium.

Conditions 1,2 , and 4 all point to one conclusionnamely, that temperature must be very carefully controlled.

\section{Description of Apparatus}

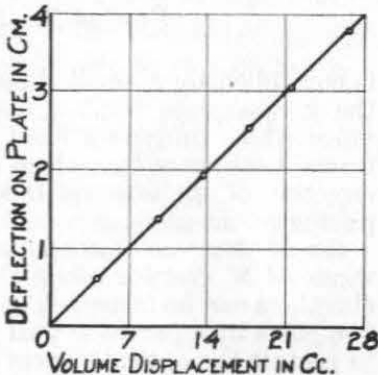

Figure 6. Calibration Curve for Pressure Unit

The apparatus (1) finally constructed depends upon the principles just discussed. It consists of six sedimentation units, each equipped to measure and record the change in pressure at the bottom of the column. These units are in one housing, using a common source of time axis movement, a common liquid supply and removal system, and a common system of temperature control. A schematic diagram of the arrangement is seen in Figure 4 .

Each unit consists of a glass-walled sedimentation tube $S$, which contains a stirring rod $S R$. The tube is closed at the bottom by a brass casting, containing a small pressure-measuring orifice. This is connected to the pressure cell $P$ by means of the tube $T$. The connection can be opened or closed by means of the valve $V$, whose operating handle projects out through the top of the case. The pressure cell $P$ is composed of a rigid outer case containing a metal bellows $B$. The change of pressure acting on the closed bottom of the bellows causes it to move. The pressure due to the normal column of liquid is counterbalanced by the mass $C B$ which is fastened to the bottom of the bellows. The movement of the bellows is indicated by the tilting of the mirror $M_{1}$, to which are fastened three needle-point supports. One, $N_{1}$, bears on a glass surface on the top of a pedestal fastened to $C B$ and therefore to the bottom of the bellows. The other two, $N_{2}$, rest on a bracket which is fastened rigidly to the case of $P$, and therefore has no movement. The tilting of the mirror is recorded by a beam of light, whose source is a small globe $E$. This is focused on the slit $S L$ by means of the lens $L_{1}$. The beam passes through the lens $L_{2}$, to the mirror $M_{1}$, then back through $L_{2}$ which brings it to a sharp focus on the lantern slide plate $L S$, housed in a light-tight plate box $P B$. The plate rests in a carriage which is given a motion perpendicular to the plane of the paper through the medium of the clock shaft

the pressure on unit area at the base would be equal to the weight of the column above it, which would be composed of the weight of the liquid plus that of the suspended particles. When the particles settle down past the point of pressure measurement, the pressure decreases. The decrease is only equal to the apparent weight of the particles suspended in $C$ and a train of gears. The operating range of the beam of light is shown by the dotted lines $L R_{1}$. For lower pressures the ray travels forward toward the settling tube $S$. As it leaves the front edge of the photographic plate it passes onto the mirror $M_{2}$, which reflects it onto a small strip of ground glass $G$ visible from the front of the apparatus. The position of the ray in this state is shown by the dotted line $R_{2}$. The intensity of the light 


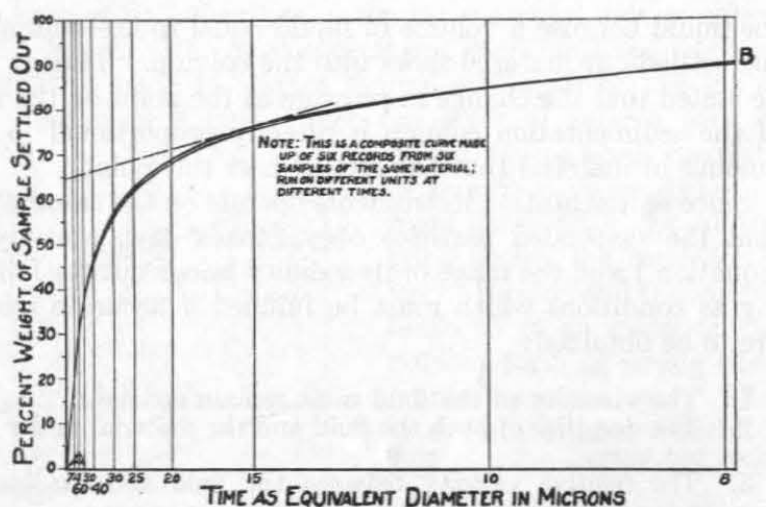

Figure 7. Analyzing Screen

is controlled by a small rheostat and milliammeter located near the ground-glass window $G$. A stationary mirror is also provided which projects a fixed light spot on the plate. This spot traces a reference line which defines both the zero value and the direction of the abscissa independent of the rough edge of the photographic plate.

Six of these units are housed in one case which has a set of windows $W$ running along the entire front, so that the settling chambers can be inspected at all times. The case is divided into two parts by a partition wall $P W$, which comes down low enough to protect the optical system from too much stray light. At the upper part of the sedimentation compartment there is an air inlet $I D$ running the entire length of the case, with numerous ports to supply air. The corresponding outlet duct $O D$ is in the lower right-hand corner of the case. These ducts are connected with a blower and a heater box outside the case. The electric heater units are controlled by a sensitive thermoelement located inside close to the sedimentation tubes. Thus the entire ease is operated as an air-bath thermostat. Figure 5 is a photograph of the apparatus as it appeared before installation.

The clock shaft $C$ runs along the entire length of the case and drives the carriages of all the units. The power is supplied by a small synchronous motor which furnishes a reliable as well as a convenient means of obtaining the movement of the time axis.

Liquid System. In the operation of the apparatus it is necessary to have a supply of the liquid at the correct temperature and also a removal system to take care of the liquid

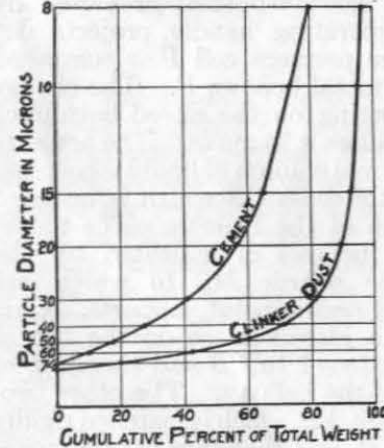

Figure 8. Method OF Plotting Size Distribution DATA

Partiele diameter $v 8$, cumulative per cent of total weight in the sedimentation chambers after analyses are completed. To meet this first requirement a thermostated storage tank is provided. The tank is arranged so that air pressure can be supplied to it and a system of piping carries the liquid to each sedimentation chamber. Thus by opening a valve the desired unit can be filled with fresh liquid. The used liquid is removed by applying suction to the hollow stirring rod. It is then stored in settling tanks for several days, after which the nearly elear liquid passes though a filtering system and is returned to the thermostated tank for further use. Care is taken to remove any traces of moisture introduced by the sample. A careful watch is maintained to see that no trace of flocculation appears in any of the units, and a suitable deflocculator is added as necessary to maintain the concentration well above the limit of safety.

Accessories. The necessary instruments for standardizing the liquid and for determining the density of the samples are located inside the case because of the desirability of making these determinations at the working temperature. They are so arranged that the measurements can be made from the outside. The viscosity is measured with a capillary tube pipet. The densities of the liquid and of the samples are determined with the help of an analytical balance mounted on the left end of the case. A fine wire is carried down from one pan into the inside of the apparatus. Liquid densities are obtained by means of an Invar plummet suspended from the wire and immersed in a beaker of the liquid. Sample densities are obtained by hanging a small bucket from the wire. A small amount of the material is placed in the bucket and weighed. The bucket is then immersed in the beaker of the fluid, the density of which has just been determined, and weighed again. Obviously, the sample density can be calculated from these two weights. A very necessary precaution in this procedure is to insure that no air is trapped in the sample when it is weighed in the liquid.

In order to facilitate operation, the apparatus is located in a dark room. This makes the changing of the plates a very simple matter, and facilitates temperature control because
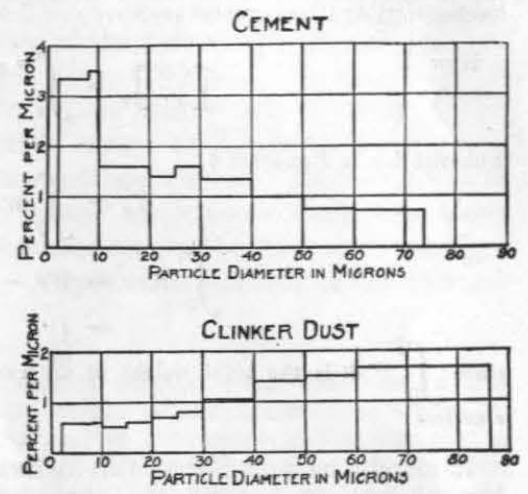

Figure 9. Method of Plotting Size Distribution Data

Method most employed in representing the entire room is used as a secondary thermostat and its temperature is held constant within $\pm 0.5^{\circ} \mathrm{F} .\left( \pm 0.3^{\circ} \mathrm{C}.\right)$

\section{Calibration and Operation}

Several factors govern the choice of the liquid to be used in the sedimentation. Most of these depend upon the sample itself; therefore a suitable liquid must be selected for each class of material. The sample and the liquid must have no chemical reaction, the liquid must not induce floceulation of the sample, and the rate of fall of the larger particles of the sample must be lower than the value at which Stokes' law ceases to apply. Arnold (2) found that the maximum allowable radius is

$$
r_{\max }=\frac{0.6 \eta}{v \sigma_{m}}
$$

Combining this with

$$
\boldsymbol{V}=\frac{2 g\left(\sigma-\sigma_{m}\right) r^{2}}{9 \eta}
$$

and solving for viscosity

$$
\eta_{\min }=\sqrt{0.37 r_{\max } \cdot{ }^{3} g \sigma_{m}\left(\sigma-\sigma_{m}\right)}
$$

This is the minimum allowable viscosity for the liquid to be used with a sample whose largest particles have a radius $r_{\max }$. Care must be taken to stay well over this limit.

It is necessary to know how much the beam of light deflects because of a given pressure change in the sedimentation chamber. This is easily and accurately determined by suspending a series of metal rods of known volume in the sedimentation liquid, and measuring the deflection produced by each in turn. The displacement of a given volume of liquid increases the head, or pressure, at the measuring orifice a definite amount. The calibration obtained in this manner is an over-all one, and thus includes all errors in the pressure element, optical system, etc. Figure 6 is an actual calibration curve for a unit. The deviations are so small that a 
straight-line calibration may be assumed. With this value determined it requires simply routine calculation to secure the sample weight for each unit so that the pressure ordinate on the plate will have the desired scale. Likewise the position of the initial liquid level is calculated so that when the sample is added the recording spot will fall on the plate. When this initial level is known the corresponding position of the point of light, which under these conditions appears
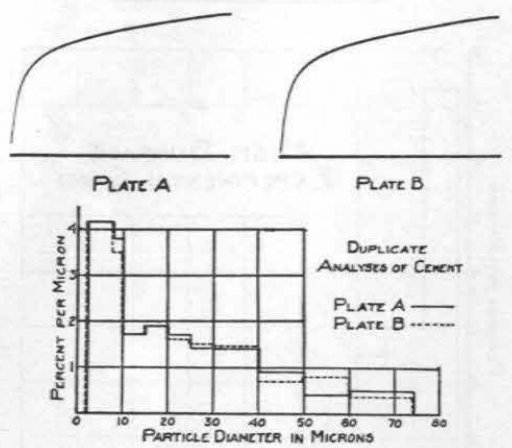

Figure 10. Duplicate Analyses of Cement on the ground-glass window $G$ (Figure 4) is marked and this mark is later used to indicate the correct level when the sedimentation chamber is filled with fresh liquid. This is a very accurate method of setting the initial level, for the spot of light is sensitive to about 3 drops of liquid.

Operation of Apparatus. The sedimentation chamber is cleaned from the last run, refilled with liquid to the correct level, and allowed to establish equilibrium.

The liquid level height is readjusted by means of a medicine dropper until the spot of light is at the correct position on the front ground glass. The light is then turned off.

The room is darkened and the plate carriage is loaded with the correctly labeled plate. The plate box cover is replaced and the rest of the operation is carried on with normal illumination in the room.

The valve between the sedimentation chamber and the pressure unit is closed and the sample is emptied into the chamber.

The sample is stirred vigorously.

The plate carriage gear train is then thrown into mesh and the light in the optical bench turned on

A few more strokes are made with the stirring rod and the valve is opened immediately after the completion of the last stroke. The stirring rod remains in position throughout the run.

Nothing is disturbed for 3.25 hours. At the end of this time the recording light is turned off, the valve is closed, the photographic plate is removed, and the unit is ready to be cleaned for the next run.

The position of the movable spot on the ground-glass window before the sample is added is determined so that the addition of the calculated weight of sample causes this spot to coincide in position with the stationary reference spot at the instant the run commences. The reference line, therefore, locates the origin of the curve, and is used for that purpose during the analyzing process.

Analysis of Plates. After development, the plates are analyzed by the tangent method previously discussed. To increase the speed of this work a projector is employed which projects the image of the curve, magnified about 4 diameters, upon the under side of the opal-glass screen. The plate may be moved in directions parallel to the two axes by means of the adjusting rods seen projecting up through the top. The screen is ruled as shown in Figure 7. The $y$ axis is graduated to show per cent settled out, since this is directly proportional to change in pressure. The $x$ axis is of course the time axis, but is graduated as diameter in microns, using the relation of Equation 3, $r=K / \sqrt{T}$.

The technic of analysis is as follows: A plate is placed in the projector, its image appearing on the screen as in $A-B$ (Figure 7). The position of the plate is then adjusted until the zero reference line is parallel to the abscissa and the upper edge of this reference line passes through the origin on the screen. The $y$ intercepts of the tangents to the top edge of the curve at the points of intersection of the micron lines are then obtained and recorded on the analysis sheet. These values with their corresponding diameters give directly the familiar cumulative weight or size table.

Since all the constants of the Stokes' law equation are determined in the routine operation of the apparatus, the theoretical value of $K$ may be readily calculated. However, Stokes' law assumes spherical bodies, and the grains in a powdered substance deviate considerably from this assumption. Therefore, it is desirable to make an experimental determination of $K$. Briefly, this was done by carefully settling out a very small fraction of material, all the particles of which had a uniform settling rate, and measuring the average diameter of these particles by the microscopic method (5). This was repeated for three other sizes of particles. The separation was made by repeated sedimentations in one of the tubes of the apparatus with standard conditions of viscosity and density. The results are shown in Table I.

Table I. Experimental Determination of $K$

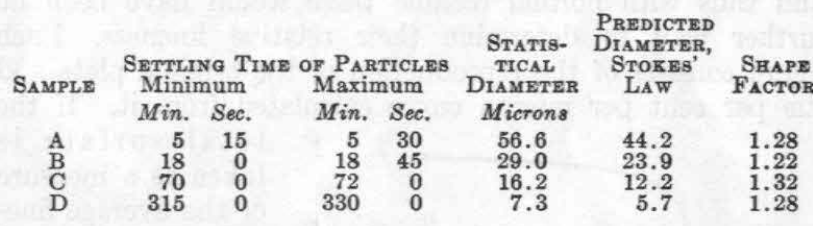

It is interesting to note the agreement between $A$ and $D$, the largest and the smallest fractions, and also that the average of the four is 1.275. An additional factor was considered before finally determining $K$. The results from the apparatus were to be correlated with the regular standard sieve analysis of the larger particles in order to obtain the complete size distribution of the sample. Screen sizes are given as the dimension of the square opening between the wires. Therefore, the microscopic determination was also made of the average size of particle just passing a standard sieve. It was found that the ratio of the microscopic diameter to the screen opening gave an average value of 1.22. Since sieve sizes are so well established, it was decided to reduce the results from the sedimentation analysis to the sieve size basis. Thus Equation 3 has the following forms:
(a) Calculated from the physical constants
(b) Determined by experiment
$D=\frac{105}{\sqrt{T}}$
(c) The latter reduced to sieve size basis
$D=\frac{134}{\sqrt{T}}$
$D=\frac{110}{\sqrt{T}}$

(c) is the form of the equation used in final analysis. Note the small difference between $(a)$ and $(c)$.

Sample Analyses. There are many graphical methods of plotting size distribution data. Figures 8 and 9 show two alternative schemes. Figure 8 is the familiar cumulation per cent $v s$. diameter curve, which is convenient for many 
purposes but is not very suitable for studying the amount of material of given size present. Figure 9 illustrates a more useful diagram, and is the one most employed in representing the Microneter data. In constructing this form of curve one precaution must be taken: the size increment must be kept the same over the entire range of diameters. If this is not done the curve shape is very misleading. It will be noted from Figure 9 that apparently the minimum diameter is 2 microns. This is a statistical minimum-i. e., a study of some special 30 -hour plates showed that there was no significant fraction of this particular material that had a diameter less than 2 microns. Figure 10 shows duplicate analyses of portions of the same cement, and demonstrates the consistency of the results obtained with the Microneter.

Figures 11 to 13 illustrate the great variation in size distribution found in fine powders. Each represents the analysis of a different sample of material. However, all the samples passed 100 per cent through the 200 -mesh sieve and thus with normal technic there would have been no further way to determine their relative fineness. Each figure consists of the reproduction of the original plate and the per cent per micron curve calculated from it. If the

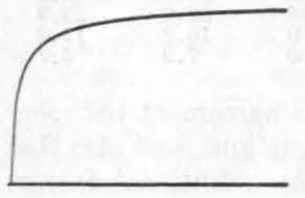

Low Surface
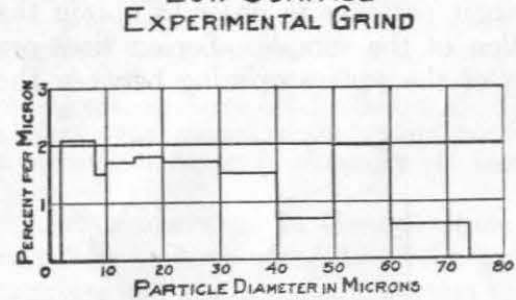

Figure 12. Low Surface ExperiMENTAL GRIND total surface is taken as a measure of the average fineness, the sample of Figure 13 is about four times as fine as that of Figure 11. These curves illustrate the importance of obtaining data on the size distribution of such materials, because this large difference in surface implies great differences in energy required for grinding, in speed in subsequent physical or chemical reaction, and in many properties of interest to the producer or user of such products.

\section{Conclusions}

The apparatus described has been in operation for several years, during which time it has been used to analyze more than five thousand samples. The capacity, 36 samples a 24-hour day, is great enough for work where more than the occasional sample has to be analyzed. Where the maximum capacity is not necessary, it has been found that one skilled operator can complete 18 analyses per 8-hour day, including preparing the sample, operating the machine, developing the plates, analyzing them, and calculating the results. The accuracy of these determinations of subsieve sizes is at least comparable to, if not considerably better than, that obtained with standard sieves of 200-mesh and coarser which are universally employed to analyze materials of larger particle diameters.

The apparatus has demonstrated its adaptability for use with a wide range of substances. Although designed pri- marily to analyze cement samples, it has been successfully employed for materials ranging in specific gravity from 2.7 to 3.7 , including cement clinker, cement rock, diorite, limestone, silica, sand, and slag.

Experience has demonstrated that the optimum size range for one plate is about 10 -i. e., the largest particles in the sample are about 10 times the diameter of the smallest size for which a reading is desired (but not necessarily the smallest particles present). There is no reason, however, why the position of this range cannot be varied over wide limits by proper control of the viscosity of the liquid
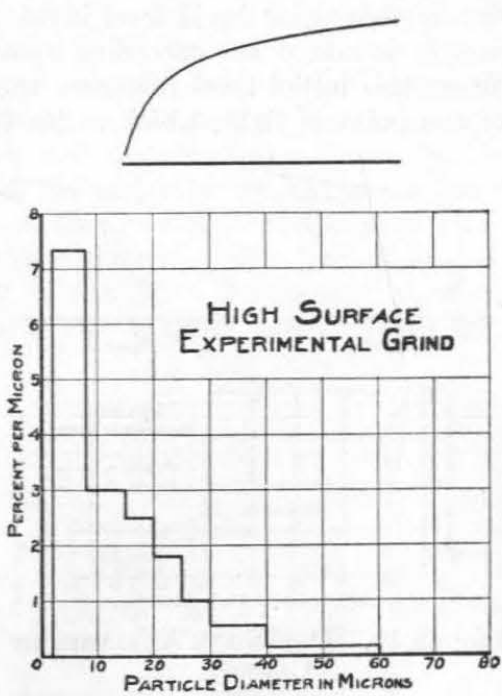

Figure 13. High Surface ExperiMENTAL GRIND

and the density difference. The practical upper limit of size is controlled by the possibility of securing a uniform mixture, which becomes increasingly difficult as the viscosity is raised. The lower limit is governed by the allowable length of time for a run, and by the entrance of colloidal effects, such as Brownian movements.

The Microneter has satisfactorily met the five specifications originally set up as the prerequisite qualities needed for a successful instrument.

\section{ACKNOWLEDGMENT}

The writer wishes to acknowledge the whole-hearted support given by John Treanor, president of the Riverside Cement Company, and by Hubert Woods, director of the Research Department of the same company, for which this work was undertaken.

Albert E. Myers assisted greatly during the early stages of development. Emory L. Ellis operated the instrument for many months, completing several thousand analyses, and put it on a producing basis. Howard Starke, who has had complete charge of the instrument for the past three years, has contributed many improvements in construction and operating technic which have decreased the labor required while increasing the speed and reliability.

\section{Literature Cited}

(1) Knapp, R. T., U. S. Patent $1,838,628$, assigned to Riverside Cement Co.

(2) Millikan, R. A., "The Electron," p. 93, Univ. of Chicago Press, 1917.

(3) Oden, Sven, Kolloid-Z., 18, 5-45 (1916); 26, 5-100 (1920).

(4) Wiegner, G., Landw. Versuchsanstalt, 91, 41 (1918).

(5) Work, L. T., Proc. Am. Soc. Testing Materials, 28, 771 (1928).

Rzcerved September 19, 1933. Presented at the Applied Mechanics Meeting, American Society of Mechanical Engineers, Pasadena, Calif,. Janusry, 1933. Published by permission of the Riverside Cement Company, Los Angeles, Calif. 